\title{
EL SEGMENTO ECOHEDÓNICO SOCIAL DE LA ISLA SANTAY DE ECUADOR (HUMEDAL 1041 RAMSAR) Y SU DISPONIBILIDAD A PAGAR (DAP) PARA SU CONSERVACIÓN
}

\author{
THE ECO-HEDONIC SOCIAL SEGMENT OF THE ISLAND OF SANTAY, \\ ECUADOR (WETLAND 1041 RAMSAR) AND ITS WILLINGNESS TO PAY \\ (WTP) FOR ITS CONSERVATION
}

\author{
Judith Genoveva Quelal Mora \\ Universidad de Guayaquil, Ecuador \\ Jenny Elizabeth Medina Alvarado \\ Universidad de Guayaquil, Ecuador \\ José Rivera Medina \\ Universidad de Guayaquil, Ecuador
}

Aceptado: 10 de julio de 2020

\section{RESUMEN}

La isla Santay, ubicada frente a la ciudad de Guayaquil, Ecuador, es un humedal de relevancia internacional RAMSAR, en la que ha disminuido el número de visitantes debido a su falta de mantenimiento. Son objetivos, del presente trabajo, encontrar la disponibilidad a pagar (DAP) por parte de los visitantes a este humedal y evaluar la posibilidad de realizar actividades de entretenimiento para familias ecohedónicas. Para ello, es necesario analizar el perfil del visitante actual que llega a la isla Santay e identificar las variables que condicionan la DAP del visitante con predilección por estos destinos turísticos, pero con mejor conservación. El método de investigación tiene enfoque positivista (cuantitativo), método descriptivo y bibliográfico. En la recolección de datos se usó la técnica de análisis de documentos y listas de cotejo.

Se analiza el perfil del visitante actual a la isla Santay y las variables que condicionan la DAP. El segmento ecohedónico es el más representativo. Sin embargo, 49\% no está dispuesto a pagar por la oferta poco variada y su precio. Asimismo, se analizan los casos de estudios de segmentación y motivaciones en ecoturismo mediante la revisión de literatura científica. Los resultados son la base para los actores del turismo que consideran unir el segmento eco-hedónico-social con los paquetes de viajes.

Palabras clave: ecohedónico social, disponibilidad a pagar (DAP), método de valoración contingente, humedal, isla Santay.

Este es un artículo Open Access bajo la licencia Creative Commons AtribuciónNoComercial-Compartirlgual 4.0

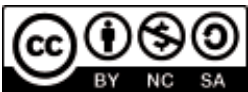




\begin{abstract}
The island of Santay, located in front of the city of Guayaquil, Ecuador, is a RAMSAR wetland of international relevance, in which the number of visitors has decreased due to its lack of maintenance. The objectives of this work are to find the willingness to pay (WTP) by visitors to this wetland and to evaluate the possibility of entertaining activities for ecohedonic families. For this, it is necessary to analyze the profile of the current visitor who arrives at the island of Santay and identify the variables that condition the WTP of the visitor with a predilection for these tourism destinations, but with better conservation. The research method has a positivist approach (quantitative), descriptive and bibliographic method. Data collection used the document analysis technique and checklists.

The profile of the current visitor and the variables that determine the WTP are analyzed. The echohedonic segment is the most representative. However, $49 \%$ are not willing to pay for the little varied offer and its price. Likewise, the case studies of segmentation and motivations in ecotourism are analyzed by reviewing the scientific literature. The results are the basis for tourism actors considering joining the eco-hedonic-social segment with travel packages.
\end{abstract}

Keywords: social eco-hedonic, willingness to pay (WTP), contingent valuation method, wetland, Island of Santay.

\title{
Introducción
}

La isla Santay es un lugar especial y vital, razón por la cual, nace el interés por conservar este importantísimo pulmón de la ciudad y sitio reconocido RAMSAR (2014):

La Convención sobre los Humedales de Importancia Internacional, conocida como la Convención de Ramsar, es un acuerdo internacional que promueve la conservación y el uso racional de los humedales. Es el único tratado mundial que se centra en un único ecosistema.

Son objetivos, del presente trabajo, encontrar la disponibilidad a pagar (DAP) por parte de los visitantes a este humedal, así como establecer la posibilidad de realizar actividades de entretenimiento, sobre todo, para niños y donde puedan acudir familias ecohedónicas. Es importante que la isla Santay se desarrolle como un lugar acogedor, tranquilo, seguro y accesible y de referencia nacional e internacional en el cuidado del medioambiente.

Por otra parte, el ecoturismo tiene una proyección de crecimiento mundial. En este contexto, las gestiones para el cuidado de la isla Santay, cantón Durán (Guayaquil, Ecuador), deben enfocarse en promocionar la visita de personas con sentido ecológico, que la cuiden, y que respeten las normas de protección del lugar; lo cual, beneficiaría a los visitantes y a los pobladores del lugar. 


\section{Estado del arte}

Ecuador es un país megadiverso, uno de los primeros en el mundo por unidad de superficie (Zambrano y López, 2015). Es una potencia turística en todos los ámbitos, pero especialmente en turismo de naturaleza. Esta megadiversidad lo demuestran sus 4 regiones naturales, 91 ecosistemas, 25000 especies de plantas (10\% del total mundial), 4500 especies de vertebrados (8\% del total mundial), 2000 especies de aves (15\% de especies endémicas del mundo), 1500 especies de mamíferos, 350 especies de anfibios, 375 de reptiles, 800 especies de peces de agua dulce y 450 de agua salada, más de un millón de especies de insectos, etc. El Ecuador, posee 53 Áreas Naturales Protegidas que representan el 19\% del territorio nacional y 12 millones de hectáreas de zonas marítimas protegidas (Galápagos). Se suman, tres hot spot o zonas calientes de diversidad para la conservación: Región del Chocó, Región tumbesina y Andes orientales, 107 áreas importantes para la conservación de las aves (IBA). Existen otros indicadores a tener en cuenta: 68\% de los turistas extranjeros quieren visitar las áreas protegidas. En 2015 llegaron más de 2 millones de visitantes a las Áreas Protegidas del Ecuador en contraste con el 2006, menos de 500 mil turistas en zonas naturales. Galápagos es el área protegida que más turistas atrae anualmente (sobrepasa el millón), su enfoque es turismo de naturaleza. En el año 2010, uno de cada 49 ecuatorianos visitaron las áreas protegidas en contraste con el año 2015, donde uno de cada 13 ecuatorianos las visitó. El turista extranjero, que visita las áreas protegidas en Ecuador, gasta 1200 dólares más que el turista promedio. Y más aún, Ecuador cuenta con 18 humedales reconocidos como sitios de importancia internacional RAMSAR (Ministerio del Ambiente del Ecuador [MAE], 2016).

Según el Plan estratégico de desarrollo de turismo sostenible para Ecuador (Plandetur, 2020), se clasifica a Guayaquil como producto 'B', Guayaquil, puerto principal y desarrollo urbano ejemplo de América. Quito y Cuenca son catalogados como productos 'A', que tiene relación con el rango de 4 a 7 noches de pernoctación de sus turistas. Existe baja participación de tour operadoras en la promoción de Guayaquil, tanto en el mercado internacional como nacional. Sin embargo, con una buena información en idioma inglés, clara, suficiente y veraz de sus destinos ecológicos, bien podría generarse recursos económicos interesantes, atrayendo exclusivamente a visitantes con interés ambiental. Se resalta además que el 92\% de los turistas nacionales y el 87\% de los turistas extranjeros recomendarían visitar la ciudad de Guayaquil. Los lugares más visitados, lo son por referencia de los habitantes, además de la información que se encuentra en Internet. Visitar las áreas naturales es una de las actividades diurnas que realizaron los turistas durante su visita a la ciudad de Guayaquil, ocupó el octavo lugar de preferencia en el primer cuatrimestre de 2015 (no hay datos recientes), según la Empresa Pública Municipal de Turismo, Promoción Cívica y Relaciones Internacionales de Guayaquil (2014, 2015).

Por otro lado, el comité ecológico de la Escuela Superior Politécnica del Litoral (ESPOL), fue el impulsor, para que finalmente la isla Santay se reconociera como sitio RAMSAR en el año 2010, lo que frenó su deforestación y uso ganadero; lo que fue ejemplo para otras organizaciones ecológicas locales y nacionales del Ecuador.

Ser sitio RAMSAR 1041 del mundo, contar con tres ecosistemas, cinco tipos de mangles y estar entre Guayaquil y Durán, identifican al humedal isla Santay. Los pobladores cuidan la isla a cambio de tener casas propias mas no el terreno en que se asientan, aun así teniendo varias limitaciones y restricciones los santileños 
viven en armonía con la naturaleza. Con el apoyo de las ONG la población santayence está disminuyendo el índice de analfabetismo y demuestra su orgullo al ser parte de la isla Santay, esforzándose cada vez más en brindar un buen recibimiento a sus visitantes.

Son escasas las investigaciones relacionadas con la segmentación en el Ecoturismo. Uno de los estudios, con 1065 encuestas, es el realizado por Díaz-Christiansen, Pérez y Ortega (2017) quiénes mediante un análisis factorial y el método de conglomerado post-hoc, analizaron las motivaciones y los perfiles de los turistas que visitan el área protegida Isla Santay en Ecuador. En esta investigación se encontraron 3 segmentos: 1.- «Los turistas sociales»: Que experimentan nuevas sensaciones con familiares y amigos y muestran las puntuaciones más bajas relacionadas a las dimensiones ecológicas. 2.- «Los eco-hedónico sociales»: Son los que obtienen las puntuaciones más altas en todos los motivos, buscan al mismo tiempo, el contacto con la naturaleza, salir de la rutina, disfrutar de amigos y familiares y obtener placer por la gastronomía. 3.- «Los eco-hedónico»: Muestran puntuaciones menores por la gastronomía y puntuaciones altas en relación a las dimensiones ecológicas. (como se citó en Carvache et al., 2018)

En consecuencia, se plantean los siguientes objetivos específicos:

1. Analizar el perfil del visitante actual que llega a la isla Santay (segmento ecohedónico).

2. Identificar las variables que condiciona la DAP del visitante para disfrutar de un destino con mejor conservación.

Surge la siguiente pregunta: ¿cuánto están dispuestos a pagar para ingresar a la isla Santay con la finalidad de conservación de la misma?

\section{Reflexiones del tema}

\section{La sostenibilidad del humedal en la isla Santay.}

La desaparición de los humedales es análogo a matar a la gallina de los huevos de oro. Todo el daño de no tenerlos se traduce en precios que continuamente se incrementan debido a la carencia de los productos. Quienes pagan por estos desastres naturales son fundamentalmente los consumidores finales, luego las empresas y el Estado. Y es que, el ambiente actúa como proveedor de vida, evidenciada en una atmósfera limpia y un mundo habitable; además de una amplia serie de servicios de amenidades que dan paso a oportunidades de actividades económicas como turismo y recreación (Freeman, 1993).

El dilema del crecimiento económico nace, según Pearce y Turner (1991), en la última cuarta parte del siglo XX, donde los impactos negativos sobre el ambiente del crecimiento económico acelerado se evidencian con el informe de la comisión Brundtland en 1987. Surge el concepto de desarrollo sustentable, aquel desarrollo que satisface las necesidades de la generación presente, sin comprometer la capacidad de las futuras generaciones, solo se piensa en satisfacer sus propias necesidades (Labandeira et al., 2007). Es necesario, por lo tanto, gestionar los recursos de manera óptima, destinándolos de forma eficaz y eficiente. 


\section{Figura 1}

Tasa de crecimiento del turismo en áreas protegidas del Ecuador entre 2006-2014 (obsérvese que no hay datos más recientes)
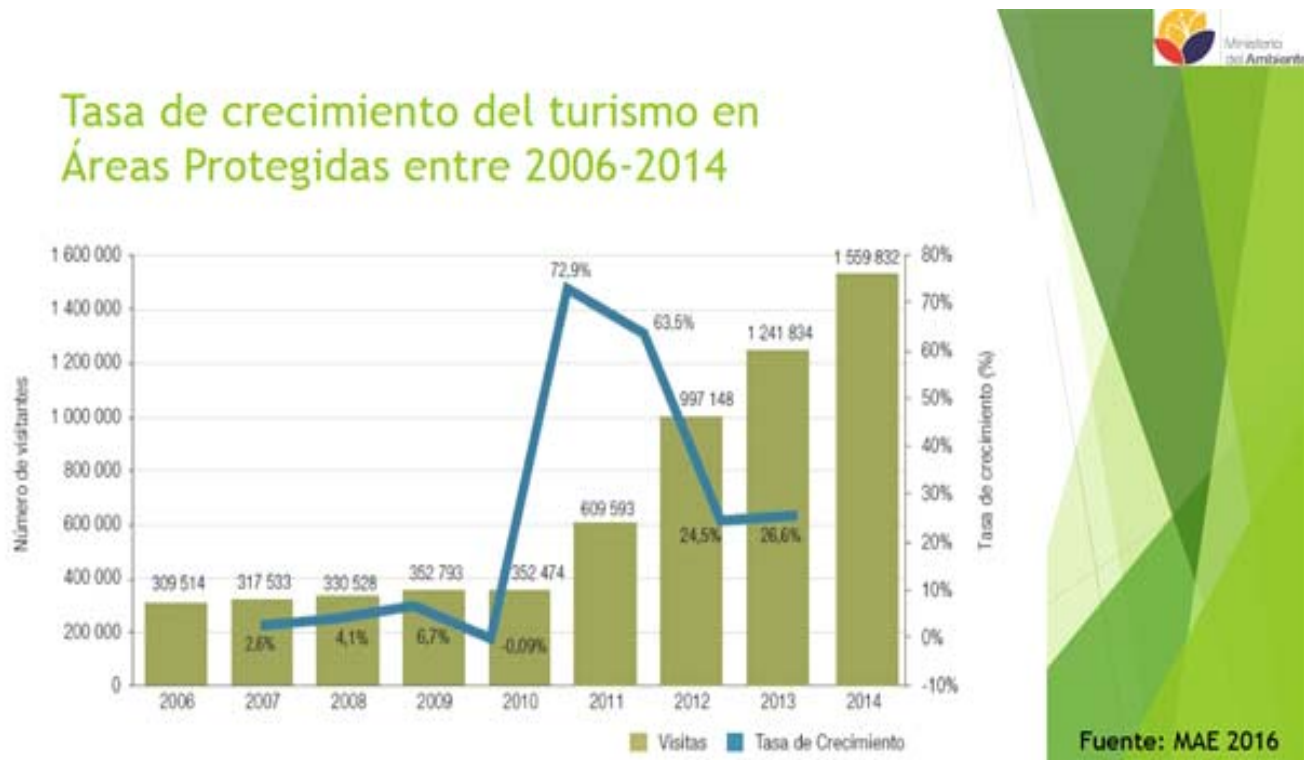

Nota. MAE, 2016.

Dentro del mercado tradicional, en pocas ocasiones, a los bienes y servicios ambientales se les otorga un precio determinado, ignorando el valor que poseen dentro del sistema económico. Esta desinformación conlleva a la naturaleza a externalidades de parte de los agentes económicos que hacen un sobreuso y aprovechamiento inadecuado, causando estas externalidades que afectan a personas vinculadas a la misma y a sus 15 generaciones futuras (Labandeira et al., 2007). Muchos de los recursos de la naturaleza, denominados bienes comunes no poseen precio de mercado, y por ende, son de acceso libre; mejor dicho, no se restringe su utilización o se limita su consumo.

Los parques nacionales son un claro ejemplo de la tragedia de los bienes comunes, ya que al estar abiertos a todo el mundo sin restricción alguna, en un área de extensión limitada y única, permite que el número de visitantes se incremente provocando el desgaste constantemente. Esta problemática, expuesta por Hardin (1968) evidencia la necesidad de un cambio de paradigma, donde los bienes comunes deben cambiar sus características o perder su valor como tales. Bajo este dilema, el autor, propone venderlos como propiedad privada (definiendo derechos de propiedad y uso) o conservar su carácter público; y que se restrinja el derecho de entrada y utilización de los mismos. Dilema que deja dos alternativas: la destrucción o la conservación de los bienes comunes que nos brindan beneficios sociales.

En esta investigación, se define al manglar como un bien común, ya que cumple con las características de no exclusión y no rivalidad del mismo; esta condición de bien común lo lleva a ser objeto de externalidades negativas al no poseer un precio determinado en el mercado, por lo que es necesario establecer su valor. 
El propósito de poner un valor monetario al medioambiente, es justificar la inversión en programas y medidas de conservación de los recursos que se cuantifican en términos monetarios; este costo debe ser cercano al valor que se le asigna al recurso aprovechado. Considerando que los recursos son escasos, debe procurarse que los beneficios de la conservación del recurso excedan los costes de la implementación de los mismos, es decir, los costos por la pérdida de los recursos o daños ambientales deben ser mayores a los costos de conservación (Pearce y Turner, 1991).

En muchos casos, la DAP no siempre es igual para todos los consumidores, por lo que hay un exceso denominado excedente del consumidor. Para medir la disposición a pagar, el precio es un indicador fundamental, pues es la representación física de las preferencias. Existen dos medidas del beneficio que se gana por mantener los recursos y dos medidas de pérdida de los mismos (Pearce y Turner, 1991):

- La disposición a aceptar prescindir de un beneficio.

- La disposición a pagar para asegurar el beneficio.

- $\quad$ La disposición a pagar para prevenir una pérdida.

- $\quad$ La disposición a aceptar una pérdida.

El turismo es sostenible solo si el turista es sostenible (Punzo, 2004). Considerando que existe una enorme cantidad de la población mundial sin conciencia ecológica, las vías de solución van en el sentido de culturizar fomentando el respeto a la naturaleza, o permitiendo ingresar a los sitios de trascendencia ecológica solo a personas amantes de la naturaleza, que paguen un costo más alto para su conservación (López, 2014, p. 228).

Por consiguiente, se requiere analizar cuál es el perfil del visitante interesado en la conservación de la naturaleza; y, más aún cuáles son las variables que condicionan la disposición a pagar del visitante para recrearse en un destino con mejor conservación.

\section{Preferencia por ecoturismo}

El ecoturismo tiene proyección de crecimiento mundial, por lo que es preciso conocer el segmento de la población que prefiere acudir a la isla Santay. En el Barómetro Turístico de Ecuador (2011), el ecoturismo y turismo de naturaleza registró $14.96 \%$ de preferencia (es el más actualizado). En este trabajo titulado Marca y hábitos del turista no residente se encuestó a 4265 visitantes en los principales puntos de salida del país durante la temporada alta.

Los segmentos mejor motivados para acudir a la isla Santay corresponden a los turistas sociales, los ecohedónico sociales y los ecohedónicos (Díaz-Christiansen et al., 2017); por lo tanto, es conveniente ofrecer actividades relacionadas con los temas de interés para estos segmentos. La generación de puestos de trabajo, gracias a los servicios brindados y productos vendidos, repercute en favor de los habitantes de la isla. Existen también algunas restricciones, por ejemplo, largas caminatas no recomendables para personas de la tercera edad. 
Agüí (1994), describe al turista que prefiere realizar ecoturismo. En líneas generales, considera que es una persona cautivada por la biodiversidad; gusta de conocer la cultura, folclor y tradiciones; contribuye con la conservación de los recursos naturales; siente agrado por el esparcimiento y el turismo alternativo. Está presto a participar en actividades recreativas, deportivas y de aventura; también a conocer la historia de la localidad, apoyar la promoción socioeconómica de las comunidades que visita y explorar la gastronomía.

La isla Santay es reconocida como un sitio RAMSAR n. ${ }^{\circ}$ 1041; ya que posee los primeros bosques de manglar del inicio del estuario del río Guayas, es un refugio para varias especies de patos, loros y otras aves migratorias incluidas en el libro rojo de aves. «Santay es el hábitat de cocodrilos de la costa (Cocodrilus acutus), especie en peligro de extinción, refugio de peces e invertebrados de agua dulce; espacios de manglar, refugio obligado para adultos y estados larvales de varios crustáceos y peces» (Ministerio del Ambiente y Agua, s.f., p. 1).

El tratado intergubernamental consagra el compromiso de Ecuador por mantener las características ecológicas de los 18 humedales que posee el país; que deben tener un uso racional o uso sostenible. El Área Nacional de Recreación Isla Santay, es considerada un área rica en biodiversidad, principalmente en lo que a presencia de aves se refiere. Florísticamente hablando, la isla es considerada como un ecosistema bastante diverso que está intervenido y en estado de recuperación. Las islas Santay y Gallo carecen por completo de bosques primarios o vírgenes, ya que se encuentran ubicadas dentro de la zona de vida correspondiente al bosque muy seco tropical.

\section{Metodología}

El método de investigación tiene enfoque positivista (cuantitativo), método descriptivo y bibliográfico. En la recolección de datos se usó la técnica de análisis de documentos y listas de cotejo.

El método de la valoración contingente (MVC), que se propone en este estudio, es una técnica de muestreo basada en la interrogación directa a personas mientras se hallan in situ, o por correo, para estimar su disposición a pagar por algo que valoran (en este caso, mejorar las oportunidades recreativas o mantener las existentes). De acuerdo al estudio de directrices de un grupo de expertos es el método empleado con mayor frecuencia para valorar el uso recreativo de los humedales templados (Barbier et al., 1997). Sin embargo, es una técnica controvertida, en parte, por el carácter de los propios valores de no uso, que se miden a menudo con su ayuda.

Se analizaron estudios de casos donde se valora el humedal isla Santay mediante el MVC y el valor económico total (VET). Adicionalmente, se encontraron las directrices para usar la valoración contingente. En la Tabla 1 se muestra un análisis comparativo de la metodología empleada en la bibliografía más relevante que se revisó, todas fueron trabajadas con el programa SPSS 22.0 y el ámbito isla Santay. Las respuestas a las interrogantes de los cuestionarios se encuentran en la Tabla 3. 


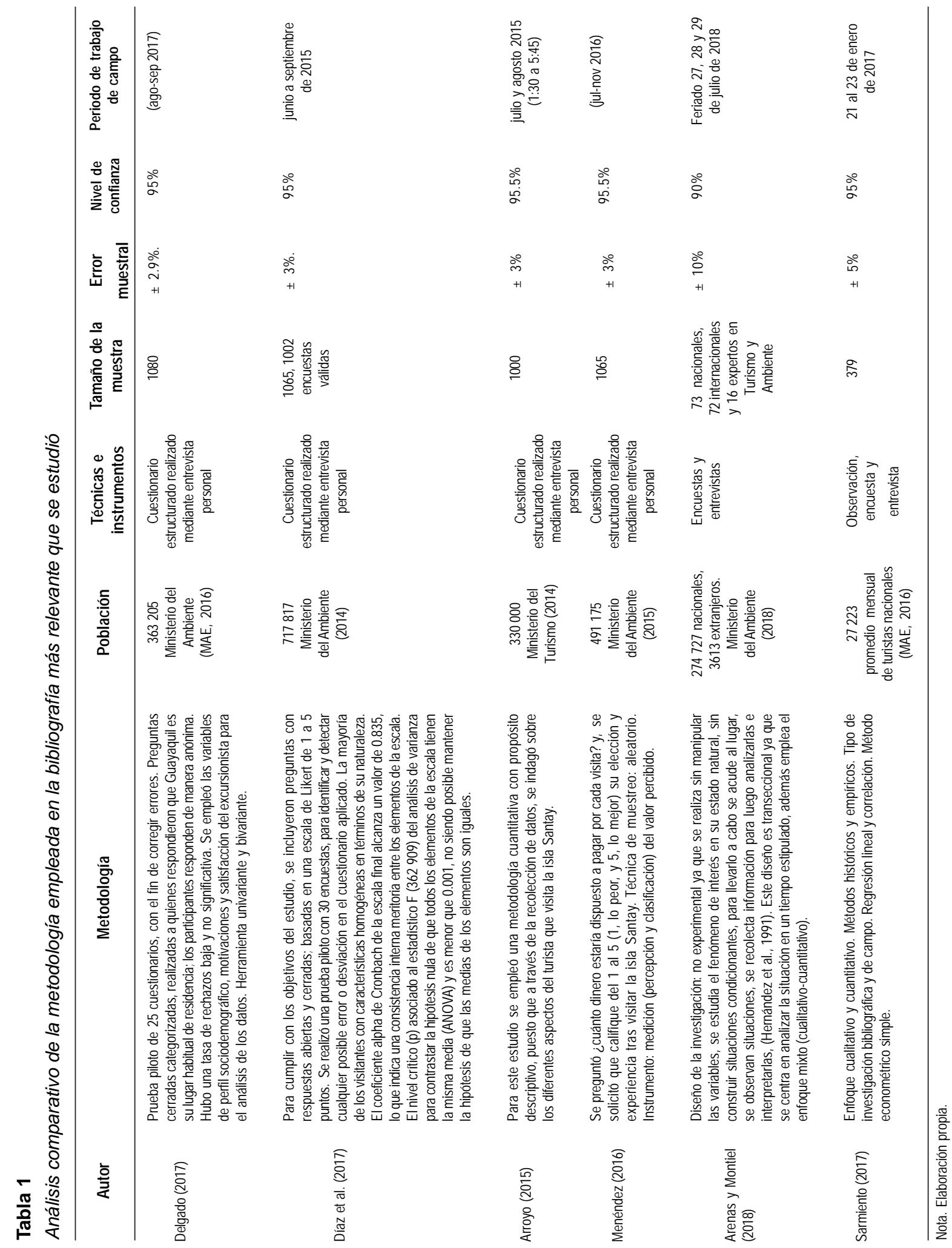


Estudio de casos

Cumpliendo con los objetivos específicos se presentan los siguientes casos de estudio:

En la Tabla 2: Segmentación de visitantes de la isla Santay, de acuerdo a un tamaño de muestra de 1065 encuestas para una población de 717818 (de las cuales 1002 resultaron válidas), se registra 83.5\% de participación que corresponde a los visitantes ecohedónico sociales; $11.8 \%$ a los turistas sociales y $4.7 \%$ a los turistas ecohedónicos. Este segmento de mayor interés, el ecohedónico social, disfruta de conocer y conectar con el medioambiente, se escapa del mundanal ruido para recrearse junto a su familia y amigos y deleitarse del arte culinario local (Díaz-Christiansen et al., 2017).

En la Tabla 3, se muestran los resultados del análisis comparativo de 6 casos de estudio entre los años 2015 y 2018. Se concluye que la mayoría que acuden a la isla Santay son mujeres con intereses ecológicos, de poder adquisitivo bajo-medio y de 18 a 35 años.

También en la Tabla 3, se aprecia que el 55\% de la muestra prefiere visitar la isla Santay entre los meses de septiembre a febrero, según estudios del periodo 2015-2019. Los meses de agosto, noviembre y diciembre son los más bajos en ventas, debido a la temporada navideña y clima en general (Morales y Zurita, 2013). Se destacan las provincias ecuatorianas Pichincha y Azuay como el $41 \%$ del turismo nacional que llega a isla Santay. En cuanto al turismo extranjero, fundamentalmente se tiene la presencia de visitantes norteamericanos, y europeos: alemanes y españoles. El ecoturismo americano (Estados Unidos, Canadá y Sudamérica) representa un 58\% del total extranjero, seguido del europeo con un 39\% y el resto del mundo con solo 3\%. Si bien el origen de las visitas pone de manifiesto que la isla Santay tiene un marcado carácter local como destino turístico natural, la presencia de visitantes, de hasta 28 países diferentes, muestra la relevancia de este enclave natural como recurso turístico para la ciudad de Guayaquil (Díaz-Christiansen et al., 2017).

En la Tabla 4, se presenta un resumen de las motivaciones para elegir un destino como Santay y trasladarse al mismo. Basicamente se asocian a dos dimensiones de motivaciones sobre la base de estudios previos adaptados a Santay. El coeficiente alpha de Cronbach de la escala final alcanza un valor de 0.835 , lo que indica una consistencia interna meritoria entre los elementos de la escala (Díaz-Christiansen et al., 2017).

\section{Disponibilidad a pagar para visitantes}

Actualmente, las personas que ingresan a la isla Santay son visitantes, no turistas, debido a que faltan lugares apropiados para hospedarse y sobre todo a la falta de agua potable. Según se aprecia en la Tabla 5, de forma desfavorable el visitante de la isla Santay, $69.1 \%$, gasta menos de \$10; además, el que tiene menores ingresos es el que más gasta, como es el caso de los visitantes que ganan menos de 500 dólares, que en comparación con los otros niveles de ingresos monetarios de los excursionistas representa el 44.2\% (Delgado, 2017).

A propósito, en la Tabla 3 se hace un análisis comparativo de la DAP, de seis casos de estudio documentados con las variables tamaño de muestra, año y lugar de estudio, donde se muestra esta disponibilidad de pago para ingresar a la isla Santay, dentro del periodo 2015-2018. 
Tabla 2

Segmentación de visitantes de la isla Santay

\begin{tabular}{|c|c|c|}
\hline$\%$ de muestra & Segmentos & \\
\hline $11.8 \%$ & Los turistas sociales & $\begin{array}{l}\text { Vivir nuevas experiencias con familiares y } \\
\text { amigos. }\end{array}$ \\
\hline $83.5 \%$ & Los ecohedónico sociales & $\begin{array}{l}\text { Además de conocer y contactar con la } \\
\text { naturaleza, se plantea la visita como una } \\
\text { herramienta para romper con su rutina, } \\
\text { disfrutar con familiares y/o amigos, y de la } \\
\text { gastronomía local. }\end{array}$ \\
\hline $4.7 \%$ & Los ecohedónico & $\begin{array}{l}\text { Bajos registros significativos en los ítems } \\
\text { relacionados con la dimensión social- } \\
\text { gastronómica. }\end{array}$ \\
\hline
\end{tabular}

Nota. Adaptado de Díaz et al., 2017.

Con el Texto Unificado de Legislación Ambiental (TULA), a partir del 2012, se dejó de cobrar \$2 nacionales y \$10 extranjeros por el ingreso a Santay y otras áreas protegidas. Adicionalmente, existe otro estudio (muestra de 2240 personas) que indica que 59.5\% de los visitantes están dispuestos a pagar por un servicio de cabañas que les permita hospedarse al menos un día en la isla Santay; de igual forma, 29\% por los servicios de recorridos guiados, 41.8\% canopy, 42.6\% torre de observación de aves y 39.8\% artesanías (Calderón y Salas, 2015). 


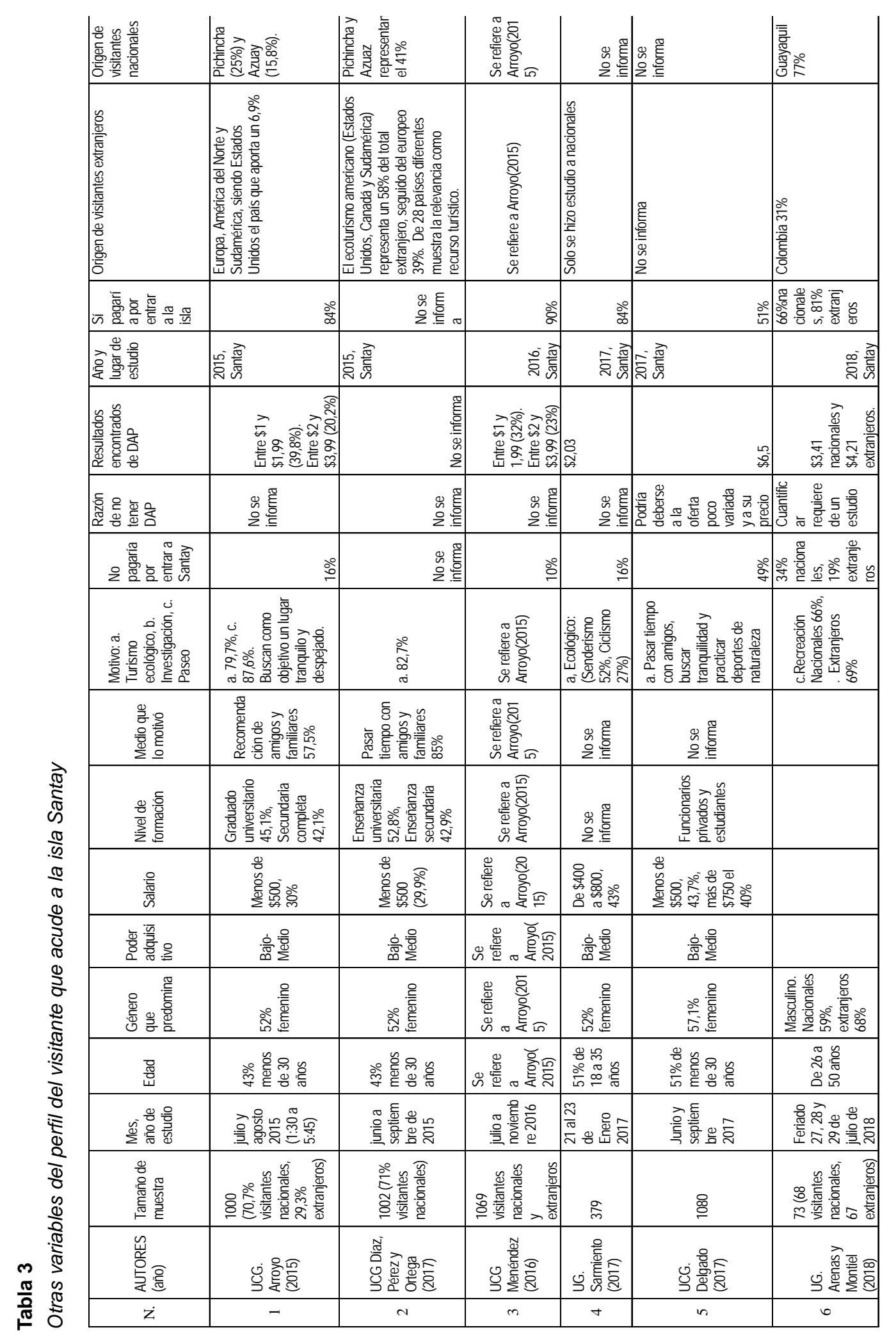


132 JUDITH GENOVEVA QUELAL MORA, JENNY ELIZABETH MEDINA ALVARADO, JOSÉ RIVERA MEDINA

Tabla 4

Matriz factorial componentes rotados. Motivación de la visita a la isla Santay

\begin{tabular}{|c|c|c|c|}
\hline \multirow{2}{*}{ Variables motivaciones } & \multicolumn{2}{|c|}{ Componentes } & \multirow{2}{*}{ Dimensiones } \\
\hline & 1 & 2 & \\
\hline Contactar con la naturaleza & 0.83 & & Ecológica-Hedónica \\
\hline Conocer su riqueza natural: flora, fauna y paisajes & 0.76 & & \\
\hline Búsqueda de tranquilidad & 0.74 & & \\
\hline Desconectar de lo cotidiano & 0.74 & & \\
\hline Una visita más de mi itinerario turístico & 0.57 & & \\
\hline Pasar tiempo con familiares y/o amigos & & 0.85 & Social-Gastronómica \\
\hline El deseo de conocer sitios nuevos & & 0.58 & \\
\hline Degustar su gastronomía & & 0.51 & \\
\hline Autovalores & 2.85 & 1.66 & \\
\hline \% varianza explicada & 35.65 & 35.65 & \\
\hline$\%$ varianza acumulada & 20.69 & 56.34 & \\
\hline $\mathrm{KMO}$ & \multicolumn{2}{|c|}{0.835} & \\
\hline Prueba de esfericidad de Barlett & \multicolumn{3}{|c|}{ Chi-cuadrado = $2134149<$ sig 0.001} \\
\hline
\end{tabular}

Método de extracción: análisis de componentes principales, método de rotación: Varimax

Nota. Díaz et al., 2017.

Tabla 5

Nivel de ingresos en dólares vs. nivel de gasto en salida a la isla Santay

\begin{tabular}{lccccccccc}
\hline \multicolumn{1}{c}{ Gasto por } & Menos de & De $\mathbf{5 0 0}$ a & De 751 a & De 1001 a & De 1251 a & De 1501 a & De 1751 a & Más de & Total \\
persona & $\mathbf{5 0 0}$ & $\mathbf{7 5 0}$ & $\mathbf{1 0 0 0}$ & $\mathbf{1 2 5 0}$ & $\mathbf{1 5 0 0}$ & $\mathbf{1 7 5 0}$ & $\mathbf{2 0 0 0}$ & $\mathbf{2 0 0 0}$ & \\
\hline Menos de $\$ 5$ & $20.4 \%$ & $6.7 \%$ & $7.3 \%$ & $4.6 \%$ & $2.2 \%$ & $1.1 \%$ & $0.5 \%$ & $0.6 \%$ & $43.4 \%$ \\
De 5 a 10 & $9.7 \%$ & $5.3 \%$ & $4.9 \%$ & $1.8 \%$ & $1.4 \%$ & $0.4 \%$ & $0.7 \%$ & $1.5 \%$ & $25.7 \%$ \\
De 11 a 15 & $3.1 \%$ & $1.4 \%$ & $1.9 \%$ & $1.0 \%$ & $0.3 \%$ & $0.1 \%$ & $0.1 \%$ & $0.2 \%$ & $8.1 \%$ \\
De 16 a 20 & $2.2 \%$ & $1.2 \%$ & $0.9 \%$ & $1.3 \%$ & $0.2 \%$ & $0.1 \%$ & $0.0 \%$ & $0.1 \%$ & $6.1 \%$ \\
De 21 a 25 & $1.0 \%$ & $0.6 \%$ & $0.8 \%$ & $0.6 \%$ & $0.1 \%$ & $0.0 \%$ & $0.1 \%$ & $0.0 \%$ & $3.2 \%$ \\
De 26 a 30 & $0.4 \%$ & $0.1 \%$ & $0.0 \%$ & $0.0 \%$ & $0.0 \%$ & $0.0 \%$ & $0.0 \%$ & $0.1 \%$ & $0.6 \%$ \\
Más de 30 & $0.9 \%$ & $0.2 \%$ & $0.1 \%$ & $0.0 \%$ & $0.0 \%$ & $0.0 \%$ & $0.1 \%$ & $0.1 \%$ & $1.4 \%$ \\
Nada & $6.3 \%$ & $0.8 \%$ & $1.8 \%$ & $1.1 \%$ & $0.5 \%$ & $0.3 \%$ & $0.2 \%$ & $0.5 \%$ & $11.5 \%$ \\
\hline Total & $44.2 \%$ & $16.3 \%$ & $17.7 \%$ & $10.4 \%$ & $4.7 \%$ & $2.0 \%$ & $1.7 \%$ & $3.0 \%$ & $100.0 \%$ \\
\hline
\end{tabular}

Nota. Delgado, 2017. 
En la Tabla 6, mediante el método VET, se analiza el beneficio anual que representa una hectárea de manglar, según precios de mercado del 2015, donde se determina que la isla Santay tiene un valor que oscila entre \$36 000 y $\$ 42000$ anuales por hectárea de manglar, a precios de mercado del año 2015, basados en el estudio de Solá (2016), con respecto a los manglares de El Morro y Churute. Con relación al valor obtenido según el método VET, se calcula el valor total unitario de una hectárea por las 2225.2 ha que representa la isla Santay.

En la Tabla 7, se registra que Santay cuenta con áreas para usos múltiples (ZUM), turismo, recreación (ZTR) y restauración (ZRE) que suman 853.63 ha; también áreas de conservación y subzona de conservación estricta que suman 1321 ha.

Tabla 6

VET de una hectárea de manglar de humedales El Morro y Churute, año 2015

\begin{tabular}{lrr}
\hline \multicolumn{2}{c}{ Beneficio del manglar en Guayas } & \multicolumn{2}{c}{ Beneficio por hectárea de manglar (\$ del 2015) } \\
\hline \multicolumn{1}{c}{ Bien o servicio } & \multicolumn{1}{c}{ Mínimo } & \multicolumn{1}{c}{ Máximo } \\
\hline Pesca & 14942.48 & 14950.84 \\
Camarón & 2279.23 & 2279.23 \\
Nutrientes & 18722.00 & 25234.00 \\
Secuestro de carbono & 280.00 & 406.00 \\
\hline Total & 36223.71 & 42870.07 \\
\hline
\end{tabular}

Nota. Solá, 2016.

Tabla 7

Distribución de la superficie de Santay

\begin{tabular}{lcr}
\hline \multicolumn{1}{c}{ Santay } & Código & ha \\
\hline Usos múltiples & ZUM & 59.03 \\
Turismo y recreación & ZTR & 96.69 \\
Restauración & ZRE & 697.94 \\
Conservación & ZCE & 1069.05 \\
Subzona de conservación estricta & & 252.09 \\
\hline
\end{tabular}

Nota. MAE, 2011. 
En la Tabla 8, se presenta el VET de Santay, según precios de mercado en 2015, de El Morro y Churute; el mínimo es de 80604999.49 y el máximo 95394 479.76; según el estudio de Solá (2016). En el caso de cobrar por el ingreso a la isla Santay, al modificar el número de visitantes, de acuerdo a los datos oficiales del MAE de 2015: De 491717 visitantes, 54\% son extranjeros y 46\% nacionales; el valor de entrada: \$10 extranjeros y \$1 nacionales. Si se cobrará la entrada el beneficio anual del humedal isla Santay por las 2225.2 ha oscilaría entre \$84 y \$99 millones.

\section{Valoración por hectárea, otros casos de estudio}

Según otros casos de estudio entre los años 1989 y 2017, bajo diferentes métodos, se valora el manglar por hectárea/año entre \$982.98 y \$57 millones (Tabla 9); la información para países en vías de desarrollo del año 2004, señala que existe un valor que oscila entre \$33 y \$57 millones anuales por hectárea/año de manglar. Hasta la quinta parte de las emisiones del mundo provenientes de la deforestación por la pérdida de manglares conllevan gran perjuicio que va desde $\$ 6$ hasta $\$ 42$ billones anuales (United Nations Environment Programme [UNEP], 2014). Dado su constante y rápido decrecimiento, los ecosistemas manglares aún existentes deben ser protegidos y gestionados de una manera sostenible para asegurar su continuidad a largo plazo y el bienestar de aquellas personas que dependen de ellos (Sarntisart y Sathirathai, 2004).

Tabla 8

VET de Santay, según precios de mercado año 2015 de El Morro y Churute

\begin{tabular}{lcr}
\hline \multicolumn{1}{c}{ Beneficio del manglar en Guayas (Morro, Churute) } & Beneficio anual por 2225.2 ha de manglar (\$ del 2015) \\
\hline \multicolumn{1}{c}{ Bien o servicio } & \multicolumn{1}{c}{ Mínimo } & Máximo \\
\hline Pesca & 33250006.50 & 33268609.17 \\
Camarón & 5071742.60 & 5071742.60 \\
Nutrientes & 41660194.40 & 56150696.80 \\
Secuestro de carbono & 623056.00 & 903431.20 \\
\hline Total & 80604999.49 & 95394479.76 \\
\hline Recreación datos de Santay (\$1 nacional y \$10 extranjero) & 4329712.00 & 4329712.00 \\
Beneficio anual Santay por 2131 ha de manglar (\$ de 2015) & 84934711.49 & 99724191.76 \\
\hline
\end{tabular}

Nota Adaptado de Solá, 2016. 
Tabla 9

Casos de estudio de valoración por hectárea de humedal

\begin{tabular}{|c|c|c|c|c|}
\hline Autor & Año de estudio & Lugar de estudio & Valor de manglar halaño & Método \\
\hline Costanza et al. & 1989 & Humedal costero de Lousiana & 982.98 a 3632.86 & $\begin{array}{l}\text { VET (valor de uso y no uso). Pesca comercial, } \\
\text { caza con trampas, recreación, protección contra } \\
\text { tormentas. Valoración total a precios de mercado } \\
\text { de } 1983 \text {, tasa de descuento del } 3 \% \text { y } 8 \% \text {. }\end{array}$ \\
\hline Costanza et al. & 1997 & América Tropical & 9.900 & \$14 785 humedal global \\
\hline Contraloría General del Estado & 1999 & Ecuador & 13061.84 & \\
\hline Ronnback & 1999 & Filipinas & $\$ 91-5299$ & Valor por pérdida \\
\hline Info. 3 RAMSAR & 2000 & Mundo & 3300 & \\
\hline Balmford et al. & 2002 & Tailandia & 60000 (manglar intacto) & \\
\hline Sarntisart y Sathirathai & 2004 & Países en vías de desarrollo & \$33 - 57 millones & Por hectárea/año \\
\hline Sola - PUCE & 2016 & Provincia de Guayas, Ecuador & 42870.07 sin recreación & VET (Valor Económico Total) \\
\hline López y Guevara (2017) & $\begin{array}{l}2017 \text { a precios de } \\
\text { mercado } 2014\end{array}$ & Urama, Venezuela & 2131 & Evaluación de impactos \\
\hline
\end{tabular}

Nota Elaboración propia.

\section{Capacidad de carga vs. demanda real}

En la Tabla 10, los casos A y B contrastan en cuanto a capacidad de carga mensual de 9000 visitantes. En el caso C, se estima 31080 visitantes mensuales, por una estadía que no supere las dos horas promedio de visita en muestra de 2240 visitantes son ecuatorianos el 97.2\%. Aproximadamente de una a dos horas es el tiempo estimado de permanencia que se emplea para recorrer la isla Santay, este indicador cumple con el tiempo recomendado de dos horas en el mismo estudio (Calderón y Salas, 2015). Otro caso de estudio, para el caso B, es una entrevista a Michael Jácome de la Empresa Pública de Parques Urbanos y Espacios Públicos, en marzo del 2013. Se expone que la isla Santay tiene una demanda potencial de 1500307 visitantes por año, sin embargo su capacidad de carga por año es de 36000 visitantes (Morales y Zurita, 2013).

También en la Tabla 10 se hace una comparación de ganancia real propuesta en dólares americanos que ingresarían a la isla Santay si se cobrara de \$1 a \$10 por entrada. Representarían ingresos mensuales totales entre \$6.467,8 y \$64.678, considerando el caso D de demanda real, según registro de entradas de visitantes (MAE, 2018). En el caso C, con una muestra de 2240 visitantes, representaría entre 31080 y 310800 dólares americanos. 
Tabla 10

Comparación de ganancia real propuesta en dólares estadounidenses

\begin{tabular}{|c|c|c|c|c|}
\hline \multirow{2}{*}{$\begin{array}{l}\text { Tabla comparativa de ganancias } \\
\text { propuestas }\end{array}$} & \multicolumn{2}{|c|}{ Valor de entrada \$ } & \multirow[t]{2}{*}{ Autor } & \multirow[t]{2}{*}{ Título del artículo } \\
\hline & 1 & 10 & & \\
\hline A. Capacidad de carga mensual & 9000 & 9000 & \multirow[t]{2}{*}{ MAE, nov., 2010} & \multirow{2}{*}{$\begin{array}{l}\text { Plan de manejo } \\
\text { ambiental }\end{array}$} \\
\hline A. Dólares ganancia & 9000.00 & 90000.00 & & \\
\hline B. Capacidad de carga mensual & 36000 & 36000 & \multirow[t]{2}{*}{ Morales y Zurita, 2013} & \multirow{2}{*}{$\begin{array}{l}\text { Plan de Marketing para } \\
\text { incentivar el uso del } \\
\text { muelle turístico de la } \\
\text { isla Santay. }\end{array}$} \\
\hline B. Dólares ganancia & 36000.00 & 360000.00 & & \\
\hline C. Capacidad de carga mensual & 31080 & 31080 & \multirow[t]{2}{*}{ Calderón y Salas, 2015} & \multirow{2}{*}{$\begin{array}{l}\text { Plan de mejora de la } \\
\text { situación actual del } \\
\text { turismo en la comuna } \\
\text { San Jacinto de Santay. }\end{array}$} \\
\hline C. Dólares ganancia & 31080.00 & 310800.00 & & \\
\hline D. Demanda promedio mensual 2018 & 6468 & 6468 & \multirow[t]{2}{*}{ MAE, mayo 2018} & \multirow{2}{*}{$\begin{array}{l}\text { Registro de visitantes } \\
\text { a mayo } 2018\end{array}$} \\
\hline D. Dólares ganancia & 6468 & 64680 & & \\
\hline
\end{tabular}

Nota. Elaboración propia.

\section{No pagaría por entrar a la isla}

No pagarían por ingresar a la isla Santay 49\% de los visitantes (Delgado, 2017), como se corrobora en la Tabla 3. A continuación se sustentan algunos motivos de acuerdo al nivel de visitas, satisfacción, mantenimiento y recurrencia.

\section{Nivel de visitas en la isla Santay.}

La curva de demanda por moda se evidenció cuando se abrieron las facilidades para visitar la isla Santay, lo que trajo un exceso de la capacidad de carga en el año 2014, alcanzando el nivel histórico más alto de visitantes mensuales de 158066 personas en octubre de ese año. Contrasta con el número máximo de visitas que podría recibir el atractivo que es de 31080 visitantes mensuales (Calderón y Salas, 2015, p. 137). La demanda de visitas disminuyó, llegando a un nivel promedio mensual de 6465 visitas en el 2018 (Tabla 11). 
Tabla 11

Nivel de visitas a la isla Santay, años 2014-2019

\begin{tabular}{lrrrrrr}
\hline Mes & 2014 & 2015 & 2016 & 2017 & 2018 & 2019 \\
\hline Enero & & 56115 & 36318 & 26622 & 4742 & 10401 \\
Febrero & & 45364 & 31243 & 21983 & 3791 & 9472 \\
Marzo & & 42086 & 35431 & 23091 & 4164 & 11380 \\
Abril & & 43377 & 24719 & 28306 & 6334 & 9475 \\
Mayo & & 29630 & 21417 & 25118 & 6008 & 10080 \\
Junio & 108232 & 28508 & 19975 & 24341 & 9164 & 10668 \\
Julio & 134871 & 41404 & 34986 & 39992 & 22154 & 14613 \\
Agosto & 107615 & 65113 & 36827 & 37308 & 19690 & 18104 \\
Septiembre & 91207 & 33017 & 27324 & 25070 & 10655 & 13990 \\
Octubre & 158066 & 46397 & 35755 & 13726 & 12435 & 9119 \\
Noviembre & 64053 & 38554 & 35992 & 6890 & 11106 & 11979 \\
Diciembre & 42345 & 22152 & 23218 & 5893 & 8088 & 8999 \\
\hline Total & 706389 & 491717 & 363205 & 278340 & 118331 & 138280 \\
\hline
\end{tabular}

Nota. MAE, 2019.

\section{Satisfacción.}

En la página web de Tripadvisor (Tabla 12), por lo general existe un alto nivel de satisfacción, con resultados de excelente: 44\%, muy bueno: 37\%, regular: 15\%, malo: 2\% y pésimo: 1\% en el periodo 2015-2019. Las personas insatisfechas comentaron:

- Los desayunos no son buenos, todo es frito sobre frito y con demasiada mantequilla.

- No hay cajero cerca y no aceptan tarjetas de crédito, por lo que hay que llevar efectivo.

- $\quad$ Se requiere llevar refrescos, bocaditos, snacks, agua.

\section{Mantenimiento.}

En el año 2018 ya se observaba un nivel bajo de visitas (Tabla 11), por lo que es necesario mejorar el mantenimiento para tener un flujo de visitantes mayor, pero que acudan siguiendo su predilección por la naturaleza y no por las tendencias de moda. Según Tripadvisor los mayores problemas relacionados al mantenimiento son los siguientes:

- Senderos con huecos que podrían ocasionar graves accidentes a los visitantes y habitantes de la isla Santay.

- Cascos poco higiénicos que se entregan con las bicicletas.

- Cocodrilos mal atendidos. 
Tabla 12

Grado de satisfacción según época del año, del periodo febrero 2015 a febrero 2019

\begin{tabular}{lcccccc}
\hline \multicolumn{1}{c}{ Época del año } & Excelente & Muy bueno & Regular & Malo & Pésimo & Total \\
\hline marzo - mayo & 33 & 25 & 12 & 3 & & 73 \\
junio - agosto & 38 & 30 & 16 & 1 & 1 & 86 \\
septiembre - noviembre & 43 & 35 & 15 & 2 & 3 & 98 \\
diciembre - febrero & 41 & 41 & 11 & 2 & 0 & 95 \\
Todo el año & 155 & 131 & 54 & 8 & 4 & 352 \\
& $44 \%$ & $37 \%$ & $15 \%$ & $2 \%$ & $1 \%$ & $100 \%$ \\
\hline
\end{tabular}

Nota. Elaboración propia.

La observación realizada en el Área Nacional de Recreación Isla Santay permitió apreciar el estado en que se encuentra la infraestructura. Se evaluó camineras, puente, centro de interpretación, ecoaldea, cocodrilera, baños, señalética y el hostal. La mayor parte de ellos se encuentra en buen estado de conservación, exceptuando los senderos que al no recibir mantenimiento frecuente van perdiendo tablones, lo cual representa peligro para los visitantes (Arenas y Montiel, 2018).

\section{Recurrencia.}

Los que visitan por primera vez la isla Santay representan el 38.52\%, por segunda y tercera vez el 32.87\% y por más de tres veces el $28.61 \%$. Se cumple la teoría de utilidad marginal, es decir, quienes visitan por primera vez son la mayor representación (Delgado, 2017).

\section{Presupuesto actual para gastar en el paseo a la isla Santay}

En la Tabla 13 se evidencia que $11.48 \%$ de las 1080 personas encuestadas, no gasta nada en sus tres primeras visitas a la isla Santay. La mayoría de los visitantes gastan lo mínimo en su visita a la isla Santay (Tabla 5).

En la Tabla 14 se presenta el presupuesto del visitante, de acuerdo al valor en transporte fluvial, alquiler de bicicletas y alimentación. Considerando el perfil del visitante (Tabla 3), al seleccionar el servicio de transporte fluvial para adulto y alimentación (no se considera alquiler de bicicletas por falta de mantenimiento de las camineras), el nivel de gasto está entre \$8.5 y \$12. En la Tabla 13 los visitantes que gastan entre \$5 y \$15 representan el 33.33\% de la muestra.

Cabe indicar que el servicio de transporte fluvial, tenía un precio de \$12 hasta enero de 2012 y luego en febrero del mismo año bajó a \$8. La cooperativa Palmeras de Santay registró una utilidad de 3327.13 en el año 2012, consecuentemente se reportaron márgenes mensuales de utilidades del 6\% al 39\%, con un promedio mensual de 25\% (Morales y Zurita, 2013). 
Tabla 13

Recurrencia de visitantes por nivel de gasto

\begin{tabular}{llcccc}
\hline & \multicolumn{4}{c}{ ¿Ha visitado con anterioridad la isla Santay? } \\
\hline & Primera vez & $\begin{array}{c}\text { Sí, de dos a } \\
\text { tres veces }\end{array}$ & $\begin{array}{l}\text { Sí, más de } \\
\text { tres veces }\end{array}$ & Total \\
\hline \multirow{5}{*}{ Gasto por } & Menos de \$5 & $17.04 \%$ & $13.70 \%$ & $13.33 \%$ & $44.07 \%$ \\
persona \$ & De 5 a 10 & $10.93 \%$ & $8.33 \%$ & $6.11 \%$ & $25.37 \%$ \\
& De 11 a 15 & $3.23 \%$ & $2.97 \%$ & $1.76 \%$ & $7.96 \%$ \\
& De 16 a 20 & $3.24 \%$ & $1.76 \%$ & $1.02 \%$ & $6.02 \%$ \\
& De 21 a 25 & $0.84 \%$ & $1.48 \%$ & $0.83 \%$ & $3.15 \%$ \\
& De 26 a 30 & $0.28 \%$ & $0.09 \%$ & $0.19 \%$ & $0.56 \%$ \\
& Más de 30 & $0.56 \%$ & $0.56 \%$ & $0.28 \%$ & $1.39 \%$ \\
& Nada & $2.41 \%$ & $3.98 \%$ & $5.09 \%$ & $11.48 \%$ \\
\hline
\end{tabular}

Nota. Delgado, 2017.

Tabla 14

Presupuesto en dolar estadounidense para visitar la isla Santay, año 2017

\begin{tabular}{lc}
\hline \multicolumn{1}{c}{ Detalle } & Costo \\
\hline $\begin{array}{l}\text { Alquiler de bicicletas (la distancia comprende 2.6 km hacia el centro } \\
\text { de la isla) }\end{array}$ & $\$ 4$ \\
Costo de acceso por bote adultos & $\$ 5$ \\
Costo de acceso por bote adultos mayores & $\$ 3$ \\
Costo de acceso por bote niños & $\$ 2.5$ \\
$\begin{array}{l}\text { La oferta gastronómica de la isla destaca en platillos típicos como secos, } \\
\text { patacones, bolones, arroz con menestra y corvina, cebiches, etc. }\end{array}$ & $\$ 3.50$ a $\$ 7$ \\
\hline
\end{tabular}

Nota. Adaptado de Alava y Sánchez, 2020.

\section{Análisis de resultados y discusión}

Anteriormente, se analizó el perfil del visitante de la isla Santay y se identificó las variables que condicionan la DAP. También, se determinó que el 51\% de los visitantes actuales están dispuestos a pagar por el ingreso \$6.5, sobre la base de una muestra de 1080 personas (Delgado, 2017).

La isla Santay es administrada por el Ministerio del Ambiente y del Agua, por ende, los comuneros de la isla Santay encuentran limitaciones para accionar. La isla está infravalorada por ineficiencia del mercado de bienes públicos, pues muchos de los servicios de los humedales se asumen como gratuitos. Delegar la gestión a personas 
ajenas, que no reconocen la importancia de los humedales, a diferencia de los usuarios o administradores locales comprometidos, no logra buenos resultados; las personas que de manera directa o indirecta tienen vínculo con la isla deben formar parte de la toma de decisión en la gestión (De Groot et al., 2007).

Al pagar entre \$1 y \$10, se tendría ingresos mensuales totales en un rango de \$6467.8 a \$64 678, lo que resulta infravalorado comparado con una cifra que beneficia a la sociedad de Santay en un año, que oscila entre \$80 y \$95 millones, bajo precios de mercado del año 2015, que incluye pesca, camarón, nutrientes y secuestro de carbono. Se reconoce que el ecoturismo americano (Estados Unidos, Canadá y Sudamérica) representa el $58 \%$ del total de extranjeros que visita la isla Santay (Tabla 3, caso 2,), seguido del europeo con el 39\%. Si el gasto promedio por turista fue de $\$ 1215.80$ en su estadía de ocho nochesl, nueve días, mayor a los años anteriores (Ministerio de Turismo de Ecuador, 2017), implica que hay una disponibilidad para gastar por día de \$135. Según resultados en la Tabla 11, el turista extranjero gasta un máximo de \$46; hay una diferencia de \$89 por aprovechar.

El visitante actual gusta de la naturaleza y de estar acompañado por amigos y familiares; puede viajar en bote, estar en el parque infantil, tener guianza, visitar el observatorio de flora y fauna, asistir a diferentes eventos artísticos, etc. La isla Santay tiene un área de 59.03 ha para usos múltiples (Tabla 7). Existen estudios en las universidades sobre reforestación, paneles solares, aviturismo (torre de avistamiento), granja apícola, huerto familiar, ruta fluvial, turismo comunitario, ecolodge con cabañas mejoradas y servicio de agua potable, marketing mediante la filmación de una película, entre otros; que no se han materializado aún por falta de recursos económicos y permisos (el Plan de Manejo es del periodo 2011-2016, pendiente de actualizar). Es necesario contar con profesionales santayences capacitados que se comprometan con los objetivos. Está presente el tema de las ranas toro, también como una amenaza (Cruz et al., 2020).

Se evidencia un decrecimiento de visitas mensuales en la Tabla 10 (caso D), representa una diferencia del 79\% con relación a la capacidad de carga (caso C), esto sumado a que no se seleccionó la categoría excelente en satisfacción (Tabla 12) y que la recurrencia baja, a medida que se visita la isla Santay, no solo por concepto de utilidad marginal, sino porque el segmento ecohedónico social encuentra caminos faltos de mantenimiento, (Tabla 13) y pocas opciones para realizar actividades en la isla Santay. Un buzón de sugerencias para conocer las opiniones o quejas de los visitantes es recomendable. Solo considerando el servicio de transporte fluvial, se puede ver que las utilidades disminuyen con el pasar de los años, así como el flujo de visitas (Tabla 11). Según la encuesta socioeconómica realizada en el año 2010 por MIDUVI, las principales actividades económicas son la pesca (37\%), empleado (4\%) y albañil (1\%).

Las estadísticas de nivel de visitas y actividades económicas permiten verificar que los habitantes de la isla Santay reciben menos ingresos por turismo comparado con otras actividades. Es decir, en otros rubros tienen mayores ingresos. Por lo tanto, es necesario entender que «los espacios naturales pueden contribuir como mecanismos idóneos para alcanzar el doble objetivo de mejorar el nivel de vida de sus habitantes, y satisfacer las necesidades legítimas de esparcimiento y ocio de la población» (INPIN, 2018). 
Algunas operadoras de turismo empaquetan a Guayaquil y Santay como turismo histórico y ecológico, además ofrecen servicio para dormir en la isla. Si estas empresas dejan ingresos económicos, mediante alianzas estratégicas con los santayences es posible mejorar el mantenimiento de las camineras en los senderos y el alquiler de bicicletas; progresivamente se puede dar solución a los demás problemas. Es preciso captar una parte del turismo ecológico, que anualmente sobrepasa el millón de turistas que llegan a Galápagos, y empaquetarlo con la visita a humedales respetando la capacidad de carga. Sería oportuno captar el segmento de turismo de sol y playa para empaquetarlo con Santay. Como ejemplo se tiene a España, la región de los cien humedales, que cuenta con usuarios frecuentes amantes de la fotografía, y de la flora y fauna, que captan visitantes por intermedio del segmento dominante de turismo: sol y playa (Cebrián, 2011).

Otro caso exitoso de la ayuda del sector privado en los humedales es el de Ducks Unlimited. Mediante actividades y con apoyo de los cazadores norteamericanos reunieron fondos para ayudar a los agricultores a conservar sus humedales. Esta iniciativa privada de conservación contribuye a reducir la brecha entre los agricultores y cazadores para la conservación de los humedales de pradera, donde todos pueden beneficiarse. Es así que la empresa crea un equilibrio entre el comercio, la comunidad, la cultura y la conservación.

\section{Conclusiones y recomendaciones}

Se analizó el perfil del visitante actual y las variables que condicionan la DAP, en consonancia con lo planteado en los objetivos específicos. El segmento ecohedónico es el más representativo, pero en general, ha disminuido el número de visitantes a la isla Santay por falta de mantenimiento. El porcentaje que no está dispuesto a pagar (49\%) encuentra las razones en la oferta poco variada y el precio.

La presente investigación, analiza los casos de estudios de segmentación y motivaciones en ecoturismo mediante la revisión de literatura científica. Los resultados son la base para los actores del turismo que consideran unir el segmento eco-hedónico-social con los paquetes de viajes. El objetivo es generar recursos económicos para la comunidad local y paralelamente preservar los recursos naturales mediante políticas de turismo, como el de Plandetur (2020).

Así como las islas Galápagos -producto estrella- tienen restricciones en su capacidad de carga, de igual forma, la isla Santay, no puede recibir a los visitantes sin ningún control (Tabla 10). Respetar reglas respecto del número de visitantes permitidos es valorar su conservación, promoviendo que solo ingrese quien se compromete con la existencia y sotenimiento del humedal Santay.

Se recomienda acoger y cuidar al visitante para que regrese bajo el concepto de ecoturismo leal; también, atender las observaciones de los organismos competentes antes de instalar un puente o elegir materiales para la restauración de las camineras, por ejemplo. Cada vez más se debe contar con proyectos sostenibles. En consecuencia, los ingresos económicos de los santayences pueden mejorar y tener alternativas de subsistencia con el turismo amigable con la naturaleza. 


\section{Limitaciones}

La valoración se considera en \$99 millones para 2225.2 ha de manglar de la isla Santay, a razón de \$42 000 ha/año de manglar (valores de 2015), por los rubros de pesca, camarón, nutrientes, secuestro de carbono, manglares de Churute (características similares en Ecuador). Lo que contrasta con \$127 millones para 2225.2 ha de manglar de la isla Santay, a razón de \$57 millones ha/año de manglar (valores de 2004), por rubros más amplios en otros países.

\section{Investigaciones futuras}

Como futura línea de investigación es conveniente analizar la oferta del ecoturismo en países latinoamericanos; así como promover estudios científicos, elevando los niveles de conocimientos en diversas áreas de la isla Santay. Otra línea de estudio, es la posibilidad de solicitar indemnización si el humedal quedara no apto para visitarlo. Siendo un área protegida del Ecuador es importante seguir en la búsqueda de soluciones.

\section{Referencias}

Agüí, J. L. (1994). Definiciones: turismo-turista. Papers de Turisme, 14-15, 77-89.

Alava, S. V. y Sanchez, M. G. (2020). Marketing Turístico para el incremento de visitantes en la isla Santay [Tesis, Universidad Laica Vicente Rocafuerte de Guayaquil]. Guayaquil.

Arenas, A. y Montiel, I. (2018). Valoración socioambiental a partir de la percepción del turista en el área nacional de recreación isla Santay, provincia del Guayas [Tesis, Universidad de Guayaquil]. Ecuador.

Arroyo, A. (2015). Perfil del turista que visita la isla Santay [Tesis, Universidad Casa Grande]. Guayaquil.

Balmford, A., Bruner, A., Cooper, P., Costanza, R., Farber, S., Green, R. E., Jenkins, M., Jefferiss, P., Jessamy, V., Madden, J., Munro, K., Myers, N., Naeem, S., Paavola, J., Rayment, M., Rosendo, S., Roughgarden, J., Trumper, K. y Turner, R. K. (2002). Economic reasons for conserving wild nature. Science, 297, 950-953.

Barbier, E., Acreman, M. y Knowler, D. (1997). Valoración económica de los humedales. Guía para decisores y planificadores. Gland-Suiza, Oficina de la Convención de Ramsar. https://www.ramsar.org/sites/default/files/documents/pdf/lib/lib_valuation_s.pdf

Calderón, V. y Salas, D. (2015). Plan de mejora de la situación actual del turismo en la comuna San Jacinto de Santay [Tesis, Escuela Superior Politécnica del Litoral]. Guayaquil.

Carvache, M., Segarra-Oña, M. y Carrascosa-López, C. (2018). Segmentación de la demanda y motivaciones en el ecoturismo. Identificación de Research Gaps en base a la discusión de análisis empírico. Revista Espacios, 39(45). https:// www.revistaespacios.com/a18v39n45/18394517.html

Cebrián, A. (2011). El turismo de naturaleza en Murcia: la región de los cien humedales. Cuadernos de Turismo, 27, $183-204$.

Costanza, R., Farber, S. C. y Maxwell, J. (1989). Valuation and management of wetland ecosystems. Ecological Economics, 1(4), 335361.

Costanza, R., d’Arge, R., de Groot, R., Farber, S., Grasso, M., Hannon, B., Limburg, K., Naeem, S., O’Neill, R. V., Paruelo, J., Raskin, R. G., Sutton, P. y van den Belt, M. (1997). The total value of the world's ecosystem services and natural capital. Nature 387, 253260.

Cruz, C., Herrera, I., Espinoza, S., Rizzo, K., Sarmiento, M., Rodas, N. y Lampo, M. (2020). New record of a feral population of Lithobates catesbeianus Shaw, 1802 in a protected area (Santay Island) in the Ecuadorian coast. BioInvasions Records, 9(2), 421-433. 
De Groot, R., Stuip, M., Finlayson, M. y Davidson, N. (2007). Valoración de humedales lineamientos para valorar los beneficios derivados de los servicios de los ecosistemas de humedales (Informe Técnico de Ramsar n. ${ }^{\circ}$ 3). Secretaría de la Convención de Ramsar, Gland, Suiza.

Delgado, O. (2017). Aporte económico al turismo comunitario procedente del excursionismo en la isla Santay [Tesis, Universidad Casa Grande]. Guayaquil.

Díaz-Christiansen, S., Pérez-Gálvez, J. y Ortega, M. (2017). Análisis de las motivaciones hacia el ecoturismo en Ecuador. Un estudio de caso en la isla Santay. Revista Turydes, 10(22). http://www.eumed.net/rev/turydes/22/isla-santay.html

Freeman, M. (1993). The measurement of environmental and resource values: theory and methods. Resources for the future.

Hardin, G. (1968). La tragedia de los comunes. Science, 162(3859), 1243-1248.

Hernández, R., Fernández, C. y Baptista, P. (1991). Diseños no experimentales de investigación. En Metodología de la investigación (pp. 187-206). https://www.esup.edu.pe/descargas/dep_investigacion/Metodologia\%20de\%20la\%20investigaci\%C3\%B3n\%205ta \%20Edici\%C3\%B3n.pdf

INPIN. (2018). Contextualización de la Valoración Ambiental, Externalidades positivas, Método costo viaje para Isla Santay - Durán, Ecuador.

Labandeira, X., León, C. y Vásquez, M. X. (2007). Economía Ambiental. Pearson Educación.

López, Y. (2014). Factores determinantes de la disposición a pagar por un destino más sostenible. El caso de la Costa del Sol occidental. Cuadernos de Turismo, 33, 199-230.

López, N. y Guevara, E. (2017). Valoración ambiental del Humedal Urama. Revista Ingeniería UC, 24(3), 279-289. Universidad de Carabobo. http://www.redalyc.org/articulo.oa?id=70754692002

Menéndez, C. (2016). El valor percibido de los turistas que visitan la isla Santay [Tesis, Universidad Casa Grande]. Guayaquil.

Ministerio del Ambiente (MAE). (2011). Plan de manejo Área Nacional de Recreación Isla Santay y Gallo.

Ministerio del Ambiente (MAE). (2016). Ecosistemas como estrategia de turismo. http://maetransparente.ambiente.gob.ec/documentacion/ Biodiversidad/Archivos/Ecosistemas_como_estrategia_de_turismo-Martin_Vega.pdf

Ministerio del Ambiente (MAE). (2019). Estadísticas de visitación a la isla Santay 2014-2019.

Ministerio del Ambiente y Agua. (s.f.). Niños conocieron importancia de la Isla Santay, Humedal Ramsar de Guayaquil. https://www.ambiente.gob.ec/ninos-conocieron-importancia-de-la-isla-santay-humedal-ramsar-de-guayaquil/

Morales, R. y Zurita, V. (2013). Plan de Marketing para incentivar el uso del muelle turístico de la isla Santay [Tesis, Escuela Superior Politécnica del Litoral]. Guayaquil.

Pearce, D. y Turner, K. (1991). Economics of Natural Resources and the Environment. The Johns Hopkins University Press.

Plandetur. (2020). Plan estratégico de desarrollo de turismo sostenible para Ecuador. https://jorgepaguay.files.wordpress.com/2012/ 03/plandetur.pdf

Punzo, L. F. (2004). Sostenibilidad del turismo y desarrollo económico local: El caso de la región Toscana. Cuaderno Virtual de Turismo, $4(1), 8-27$.

RAMSAR. (2014). La Convención de Ramsar: ¿de qué trata? (Ficha informativa n. ${ }^{\circ}$ 6). https://www.ramsar.org/sites/default/files/ fs_6_ramsar_convention_sp_0.pdf

Ronnback, P. (1999). The ecological basis for economic value of seafood production supported by mangrove ecosystems. Ecological Economics, 29, 252-253.

Sarmiento, M. (2017). Valoración económica de la disposición del mercado para la conservación de los recursos turísticos como herramienta de desarrollo sostenible en el área nacional de recreación isla Santay [Tesis, Universidad de Guayaquil].

Sarntisart, I. y Sathirathai, S. (2004). Shrimp farming and mangrove loss in Thailand. (Barbier, E. B. \& Sathirathai, S.) 96-113 (Edward Elgar, 2004). 
144| JUDITH GENOVEVA QUELAL MORA, JENNY ELIZABETH MEDINA ALVARADO, JOSÉ RIVERA MEDINA

Solá, D. (2016). Impacto económico de la pérdida de los servicios ambientales del manglar en Guayas [Tesis, Pontificia Universidad Católica del Ecuador].

Tripadvisor. (s.f.). https://www.tripadvisor.co/ShowUserReviews-g303845-d6874296-r681522485-Isla_Santay-Guayaquil_Guayas_ Province.html

United Nations Environment Programme World Conservation Monitoring Centre (UNEP). (2014). The Importance of Mangroves to People: A Call to Action. En E. S. J. van Bochove (Ed.) (p. 128). Cambridge.

Zambrano, R. y López, M. (2015). Breve historia y perspectivas para el futuro del Sistema Nacional de Áreas Protegidas del Ecuador (SNAP). Memorias, 42. Contribuciones Científicas. Manabí, Ecuador.

Judith Genoveva Quelal Mora

Economista con mención en Gestión Empresarial, especialización Finanzas. Maestría en Administración de Empresas. Diploma Superior en Administración de Empresas. Docente investigadora con aval de SENESCYT. Docente de la Universidad de Guayaquil desde el año 2014. Trece años de experiencia en el sector privado y público. judith.quelalm@ug.edu.ec

gquelal@gmail.com

ORCID: https://orcid.org/0000-0003-2231-4273

Jenny Elizabeth Medina Alvarado

Economista. Diplomado Superior en Gestión de Marketing. Maestría en Administración de Empresas. Maestría en Negocios Internacionales y Gestión en Comercio Exterior, especialización en Negocios Internacionales. Docente de la Universidad de Guayaquil desde el año 2010. Veintiun años de experiencia en el sector privado y público. jenny.medinaa@ug.edu.ec

ORCID: https://orcid.org/0000-0001-5877-500X

José Rivera Medina

Licenciado en Gestión de Diseño y Comunicación Visual. Maestría en Marketing y Dirección Comercial. Docente de la Universidad de Guayaquil desde el año 2010. jose.riveramed@ug.edu.ec

ORCID: https://orcid.org/0000-0001-9100-4039 0992264483 